\title{
Measuring strong magnetic fields of neutron stars with the next generation of X-ray instruments
}

\author{
G. Schönherrª ; P. Kretschmar ${ }^{b}$, J. Wilms ${ }^{c}$, I. Kreykenbohm ${ }^{c}$, F. Schwarm ${ }^{c}$, \\ K. Pottschmidt ${ }^{d, e}$, R.E. Rothschild ${ }^{f}$ \\ ${ }^{a}$ Leibniz-Institut für Astrophysik Potsdam, An der Sternwarte 16, 14482 Potsdam, Germany \\ ${ }^{b}$ European Space Astronomy Centre (ESA/ESAC), Science Operations Department, Villanueva \\ de la Cañada (Madrid), Spain \\ ${ }^{c}$ Dr. Karl-Remeis Sternwarte Bamberg, Astronomisches Inst. der Univ. Erlangen-Nürnberg, \\ Sternwartstr. 7, 96049 Bamberg, Germany \\ ${ }^{d}$ CRESST and NASA Goddard Spaceflight Center, Greenbelt, Maryland 20771, USA \\ ${ }^{e}$ CSST, University of Maryland Baltimore County, Baltimore, Maryland 21250, USA \\ ${ }^{f}$ CASS, University of California, San Diego, 9500 Gilman Drive,La Jolla, CA 92093-0424, USA \\ Email: gschoenherreaip.de
}

\begin{abstract}
To date, the only direct method to assess the strong magnetic fields of High-Mass X-ray Binaries containing accreting neutron stars is the observation and analysis of electron cyclotron resonance features (short: "cyclotron lines" or CRSFs) in their high-energy X-ray spectra. We dicuss how the upcoming era of new observatories and instrumentation like IXO will change our "magnetic" view of those objects. The science case for IXO in this respect does not focus on the measurement of the cyclotron lines themselves (which are typically at $10-100 \mathrm{keV}$ ) but on obtaining new constraints on the accreting sources' geometries and physical parameters as key ingredients to disentangle their influences and understand the overall timing and spectral properties of neutron star highmass X-ray binaries.
\end{abstract}

Fast X-ray timing and spectroscopy at extreme count rates: Science with the HTRS on the International $X$-ray Observatory - HTRS2011,

February 7-11, 2011

Champéry, Switzerland

\footnotetext{
*Speaker.

${ }^{\dagger}$ on behalf of the MAGNET collaboration
} 


\begin{tabular}{llcccl}
\hline Source & $E_{c y c}(\mathrm{keV})$ & $P_{p u l s}(\mathrm{~s})$ & $P_{\text {orb }}(\mathrm{d})$ & companion & discovery \\
\hline \hline Swift J1626.6-5156 & 10 & 15 & $23 ?$ & Be & RXTE (deCesar, '11, submitted) \\
4U 0115+63 & $14,24,36$, & 3.6 & 24.31 & Be & HEAO-1 (Wheaton, '79) \\
& 48,62 & & & & RXTE, SAX (Heindl '99, Sant.,'99) \\
V 0332+53 & $27,51,74$ & 4.37 & 34.25 & Be & Ginga (Makishima, '90) \\
4U 1907+09 & 18,38 & 438 & 8.38 & B2 III-IV & SAX (Cusumano, '98) \\
Vela X-1 & 24,52 & 283 & 8.96 & B0.5Ib & Mir-HEXE (Kendziorra, '92), \\
& & & & & RXTE (Kreykenbohm, '02) \\
A0535+26 & 50,110 & 105 & 110.58 & Be & HEXE (Kendziorra, '92, '94), \\
& & & & & CGRO (Maisack, '97) \\
4U 1538-52 & 20 & 530 & 3.73 & B0I & Ginga (Clark, '90) \\
Cep X-4 & 28 & 66.25 & $>23$ & B1 & Ginga (Mihara, '91) \\
Cen X-3 & 29 & 4.8 & 2.09 & O6.5II & SAX (Santangelo, '98) \\
& & & & & RXTE (Heindl, '98) \\
X Per & 29 & 837 & 250.3 & B0 III-Ve & RXTE (Coburn, '01) \\
MXB 0656-072 & 33 & 160 & $100 ?$ & O9.7Ve & RXTE (Heindl, '03) \\
XTE J1946+274 & 36 & 15.8 & 169.2 & B0-1V-IVe & RXTE (Heindl, '01) \\
4U 1626-67 & 37 & 7.66 & 0.028 & WD? & SAX (Orlandini, '98) \\
& & & & & RXTE (Heindl, '98) \\
GX 301-2 & 37 & 690 & 41.5 & B1.2Ia & Ginga (Mihara, '95) \\
Her X-1 & 41 & 1.24 & 1.7 & A9-B & Ballon-HEXE (Trümper, '78) \\
OAO 1657-415 & $36 ?$ & 37.7 & 10.4 & B0-B6Ia-Iab & SAX (Orlandini, '99) \\
\hline
\end{tabular}

Table 1: Overview of NS sources with detected cyclotron lines. The references given refer to the discovery of the line(s). Table: courtesy of J. Wilms (2)

\section{Cyclotron line detections}

Since the detection of the first cyclotron resonance scattering feature in the X-ray spectrum of Hercules X-1 in 1978 by Trümper et al. (1), magnetic field determinations via cyclotron lines have been made for a total of 16 accreting neutron star binaries (2). However, this number implies that still less than 1/4th of the known sample of X-ray pulsars (3) have detections of cyclotron lines, although the observational data has advanced significantly both in quality and quantity since the first detection. It is still unclear why cyclotron lines are observed in some sources and not in others, i.e., if this has to be accounted to intrinsic differences in these systems' properties or if the non-observability simply results from instrumental limitations for less prominent line features (4).

The physical process behind cyclotron lines is resonant scattering of the $\mathrm{X}$-ray continuum photons with electrons which are quantized in their perpendicular momenta due to a strong $B$-field. In a typical neutron star high-mass X-ray binary electron cyclotron lines are expected to form as absorption-like features in the $\sim 10-100 \mathrm{keV}$ range. This energy range corresponds to magnetic field strengths of the order of $10^{12}$ Gauss, as deduced from the approximate " $12-B-12$ " relation: $B\left[10^{12} \mathrm{G}\right]=E_{\text {line }}^{(n)}(1+z) /(n \times 12 \mathrm{keV})$, where $z$ is the gravitational redshift, usually assumed to be of the order of $z=0.3$ and $n=0,1,2, \ldots$ is the harmonic line number. 


\section{Probing the magnetic field structure}

The CRSF energies are directly linked to the magnetic field strength - and magnetic field geometry - of the neutron star. A key question for interpreting the measured line energies, however, remains: what is the location of the line forming region? While the local value of the magnetic field strength can vary considerably even for the assumption of a simple dipolar structure within an extended accretion column, there are unfortunately no general, hard constraints on the location and geometry of the line-forming region for accreting neutron star binaries neither from theory nor from observations. Recent modelling work seems to point towards a scenario where the observed radiation comes from a rather extended region where the superposition of the imprints from cyclotron line features would also offer a natural explanation for various observational findings such as deviations from harmonic line ratios of several line features, strongly asymmetric line shapes and could even account for the non-observability of cyclotron lines in many sources depending on the geometric setting (see, e.g., 5). In previous work (6), we have undertaken the first quantitative model-to-data comparison by fitting the simulation based XSPEC model cyclomc (Schönherr \& Wilms) to observational data. That comparison has further proven the necessity to introduce more complex geometries into our numerical simulations. As the fitting procedure in the current version of cyclomc already relies on many free parameters, the introduction of additional degrees of freedom is, however, not unproblematic. The line shapes of the scattering features that the observer sees depend heavily not only on the magnetic field geometry but also on the emission angle, i.e., on the viewing geometry which in turn is changing with the rotational (pulse) phase, which again links to the location and extension of the line-forming region (7), the temperature of the electron plasma etc. Constraints from a unified approach (timing, spectroscopy and - soon - polarimetry) are therefore strongly needed to make the computational effort of a more realistic model feasible in the future.

In the following we will discuss our expectations on what we will learn on the observational side with the upcoming new instruments to further understand cyclotron line sources and by what methods we will be able to obtain new insights in the physical setting. Especially future observatories bearing polarimetric instruments will open a new parameter space for understanding X-ray pulsars independent from and in combination with cyclotron line analyses. This will have implications onto the adaptation of our Monte Carlo simulations for the resonant photon-electron scattering process.

\section{Timing and phase-resolved spectroscopy}

As the viewing geometry on an X-ray pulsar changes with its rotational phase, timing analysis is fundamental for understanding the nature of a pulsar's X-ray emission. In addition to those variations resulting from the rotating, beamed emission from one magnetic pole, further pulsephase variability can be superimposed by the changing conditions of the observability of X-ray photons originating from either magnetic pole taking into account general relativistic light bending. Both effects imply that the observability and the shape of cyclotron lines will heavily depend on its pulse phase in a non-trivial way. From the one-dimensional momentum quantization of the plasma electrons, in principle less prominent lines are expected if the line of sight is closer to 
the magnetic axis (the observer views the magnetic pole face-on; photons escape parallel to the magnetic field axis). But even here, the spectral contributions of both poles must be disentangled a task which in principle requires knowledge also of the intrinsic beam patterns in addition to the accretion column's geometry. Therefore, one future challenge will be to find models for the pulsed continuum emission from high-precision timing and phase-resolved spectroscopy and to combine it with line formation models which can in turn provide a measure for observed phase-variations of cyclotron lines (see also the contribution by C. Ferrigno).

\section{High-resolution spectroscopy}

Simulations $(5 ; 6 ; 8)$, observations $(9 ; 10)$ and theoretical considerations agree on a complex fundamental line shape. Eventually existent fine structure might become observable through highresolution spectroscopy. Further future challenges for new instrumentation and theoretical models lie in resolving the mystery of the " $10 \mathrm{keV}$ bump" in the continuum spectra and - especially for IXO's energy range - to maybe detect signatures of proton cyclotron lines in the soft energy range.

New instruments with a better signal-to-noise ratio could lead to further electron cyclotron line detections, either increasing our source sample or detecting additional lines in known cyclotron line sources. Due to the high-energy cutoff of an X-ray pulsar's continuum spectrum, a very high signal-to-noise ratio is required for the observability of higher harmonics in general. Simulations have furthermore shown that very shallow fundamental lines - which are obviously even harder to detect - are to be expected for extended line forming regions.

\section{Polarimetry: fan or pencil beam?}

Once X-ray polarimeters are available, a new window to the physics of compact objects and in particular accreting X-ray pulsars will be opened. The fact that accreting X-ray pulsars cannot be resolved by imaging restricts their accessable parameter space so far to energy and time. IXO with its planned payload instrument XPOL would be one of three near-future X-ray observatory missions (these are: GEMS, IXO, NHXM) to include X-ray polarimeters which are suitable for the science case of compact objects.

X-rays from accreting neutron stars should be highly polarized. Measuring their polarization properties would allow incomparable insights in their beaming and radiation mechanisms and their magnetic field structure (see, e.g, $(11 ; 12)$ ). One fundamental uncertainty which could be clarified using time-resolved X-ray polarimetry is the discrimination between pencil and fan beam characteristics of the X-ray emission from the magnetic poles (see, 12, and figures 2,3 within.). As shown by Mészàros already in 1988, the polarization characteristics of an accreting neutron star binary depend in a characteristically different way on the pulse phase for these two orthogonal geometries of the emission region. Explicitly, as these authors have shown, the variation of the amount of linear polarization and the polarization angle with the pulse phase and amplitude gives a discrimination measure between fan and pencil beam patterns over the whole spectral energy range. This is an important point for its application, as the first generation polarimeters are planned to encompass only a range of $1-10 \mathrm{keV}$ which is below practically all observed cyclotron line energies. Determining this geometrical constraint for the sources of our sample would resolve a basic uncertainty in the 
physical setting and, by reducing the dependent free parameters, greatly improve the predictive quality of our quantitative fitting approach of physical models to spectra data. In order to test measured values of polarization on cyclotron lines within the complete picture, we will in the future also include polarization dependent scattering cross sections into our new simulation code $(8 ; 13)$. Polarization of the X-ray emission might also be one reason why we sometimes do not observe higher harmonics as the depths of the higher harmonic features strongly depend on the fraction of photons of certain parallel mode (14).

\section{References}

[1] J. Trümper et al., ApJ 219, L105, 1978.

[2] J. Wilms, ASTRON conf. proc., 2010.

[3] Q. Z. Liu et al., A\&A 455, 3, 1165-1168, 2006.

[4] R. Rothschild et al., White paper, US Dec. Rev., 2010.

[5] O. Nishimura, ApJ 672,2, 1127-1136, 2008.

[6] G. Schönherr et al., A\&A 472: 353-365, 2007.

[7] G. Schönherr, AIP Conf. Proc. 1248, 67-70, 2010.

[8] F. Schwarm, Diploma thesis, Univ. Erlangen, 2010.

[9] K. Pottschmidt et al., ApJ 634: L97-L100, 2005.

[10] I. Kreykenbohm et al., A\&A 433: L45-L48, 2005.

[11] M. Rees, MNRAS 171, 457-465, 1975.

[12] P. Mészàros, ApJ 324, 1056-1067, 1988.

[13] R. Araya \& A. Harding, ApJ 517: 334-354, 1999.

[14] M. Isenberg et al., ApJ 493: 154-174, 1998. 